\title{
Discriminative pattern of reduced cerebral blood flow in Parkinson's disease and Parkinsonism-Plus syndrome: an ASL-MRI study
}

Lina Cheng ${ }^{1,2+}$, Xiaoyan $\mathrm{Wu}^{1+}$, Ruomi Guo ${ }^{3}$, Yuzhou Wang ${ }^{4}$, Wensheng Wang ${ }^{2}$, Peng He ${ }^{2}$, Hanbo Lin ${ }^{2}$ and Jun Shen ${ }^{1 *}$ (D)

\begin{abstract}
Background: Accurate identification of Parkinson's disease (PD) and Parkinsonism-Plus syndrome (PPS), especially in the early stage of the disease, is very important. The purpose of this study was to investigate the discriminative spatial pattern of cerebral blood flow (CBF) between patients with PD and PPS.

Methods: Arterial spin labeling (ASL) perfusion-weighted imaging was performed in 20 patients with PD (mean age $56.35 \pm 7.56$ years), 16 patients with PPS (mean age $59.62 \pm 6.89$ years), and 17 healthy controls (HCs, mean age $54.17 \pm 6.58$ years). Voxel-wise comparison of the CBF was performed among PD, PPS, and HC groups. The receiver operating characteristic (ROC) curve was used to evaluate the performance of CBF in discriminating between PD and PPS. The relationship between CBF and non-motor neuropsychological scores was assessed by correlation analysis.
\end{abstract}

Results: PD group showed a significantly decreased CBF in the right cerebelum_crus2, the left middle frontal gyrus (MFG), the triangle inferior frontal gyrus (IFG_Tri), the left frontal medial orbital gyrus (FG_Med_Orb) and the left caudate nucleus $(C N)$ compared with the $\mathrm{HC}$ group $(P<0.05)$. Besides the above regions, the left supplementary motor area (SMA), the right thalamus had decreased CBF in the PPS group compared with the HC group $(P<0.05)$. PPS group had lower CBF value in the left MFG, the left IFG_Tri, the left CN, the left SMA, and the right thalamus compared with the PD group $(P<0.05)$. CBFs in left IFG_Tri, the left $C N$, the left SMA, and the right thalamus had moderate to high capacity in discriminating between PD and PPS patients (AUC 0.719-0.831). The CBF was positively correlated with the Mini-Mental State Examination (MMSE) and Montreal Cognitive Assessment (MoCA) scores in PD patients, while positively correlated with the MMSE, Hamilton Anxiety Scale (HAMA), Hamilton Depression Scale (HAMD) scores in PPS patients $(P<0.05)$.

Conclusion: PD and PPS patients have certain discriminative patterns of reduced CBFs, which can be used as a surrogate marker for differential diagnosis.

Keywords: Parkinson's disease, Parkinsonism-plus syndrome, Magnetic resonance imaging, Arterial spin labeling

\footnotetext{
* Correspondence: shenjun@mail.sysu.edu.cn

${ }^{\dagger}$ Lina Cheng and Xiaoyan Wu contributed equally to this work.

'Department of Radiology, Sun Yat-Sen Memorial Hospital, Sun Yat-Sen University, Guangzhou 510120, China

Full list of author information is available at the end of the article
} 


\section{Background}

Parkinson's disease (PD) and Parkinsonism-Plus syndrome (PPS) are clinically common chronic progressive neurodegenerative diseases in middle-aged and senior population. The known pathological basis of PD is over $50 \%$ reduction of dopaminergic neurons in the substantia nigra pars compacta of the midbrain, resulting in a decrease of dopamine production and a relative increase of antagonistic neurotransmitter acetylcholine, which causes hyperfunction. PPS includes a variety of diseases, such as multiple system atrophy (MSA), progressive supranuclear palsy (PSP), and corticobasal degeneration (CBD). The common feature is the implication of the extrapyramidal system [1-3]. Albeit similar symptoms, clinical treatment for PD and PPS is obviously different [4-6]. Thus, accurate identification of these two types of diseases, especially in the early stage of the disease, is very important $[7,8]$.

Previously, some brain structural changes on magnetic resonance imaging (MRI) have been shown to be able to differentiate between PD and PPS, such as a cross sign, fissure sign, hummingbird sign, swallow tail sign [9-11]. Unfortunately, these signs mainly occur in the later stage of diseases. Positron emission tomography (PET) and single-photon emission computed tomography (SPECT) can be used for early identification by observing metabolic changes in the brain region. However, it is not widely available and suffers from shortcomings of high cost and radiation hazards [12, 13]. Besides structural MRI, functional MRI has additional value for differential diagnosis [14-18]. Among these functional imaging, arterial spin labeling (ASL)-based perfusion-weighted imaging is a novel, non-invasive method that does not require the injection of contrast agent. Currently, threedimensional pseudo-continuous arterial spin labeling (3D-pCASL) has been used to measure cerebral perfusion in neurodegenerative diseases quantitatively [19] and demonstrated abnormal cerebral perfusion in PD and MSA [20, 21].

However, whether the spatial pattern of cerebral blood flow (CBF) as measured by ASL imaging is different between patients with PD and PPS remains unclear. In this study, 3D-pCASL was performed in PD and PPS patients to investigate the discriminative spatial pattern of $\mathrm{CBF}$ abnormalities in patients with PD and PPS.

\section{Methods}

\section{Subjects}

From Jan 2017 to Jan 2019, consecutive patients who were clinically diagnosed with PD and PPS were enrolled. The diagnosis of PD was confirmed according to the Clinical Diagnostic Criteria for Parkinson's Disease in China (2016) [22], and current consensus clinical criteria for PPS [2]. The exclusion criteria were as follows: cerebral hemorrhage, infarction, brain tumors, trauma, severe white matter hyperintensity, contraindication to MRI, and image quality of structural or ASL imaging ineligible for data analysis. Finally, 20 PD patients including 13 males and 7 females, with a mean age of $56.35 \pm 7.56$ years (range, 43 to 66 years), and 16 PPS patients including 7 males and 9 females, with a mean age of $59.62 \pm 6.89$ years (range, 41 to 66 years) were enrolled. 17 healthy controls (HCs) were collected, including 6 males and 11 females, with a mean age of $54.17 \pm$ 6.58 years (range, 44 to 65 years). Non-motor neuropsychological scores including Hamilton Anxiety Scale (HAMA), Hamilton Depression Scale (HAMD), MiniMental State Examination (MMSE), and Montreal Cognitive Assessment (MoCA) were obtained in PD and PPS patients. All neuropsychological evaluations and MRI were performed under medication in "on" state, similar to a previous study [23].

\section{MRI acquisition}

All participants underwent an MRI examination on a 3.0 T scanner (Signa HDxt, GE Medical Systems) with an 8-channel, phased-array head coil. The sequences included axial three-dimensional brain volume T1weighted imaging (repetition time $[\mathrm{TR}]=8.8 \mathrm{~ms}$, echo time $[\mathrm{TE}]=3.5 \mathrm{~ms}$, inversion time $[\mathrm{TI}]=450 \mathrm{~ms}$, flip angel $[\mathrm{FA}]=13^{\circ}, \quad$ Matrix $=320 \times 320$, field of view [FOV] $=240 \times 240 \mathrm{~mm}$, number of excitations $=1$, slice thickness $=1.2 \mathrm{~mm}$, slice gap $=0 \mathrm{~mm}$ ) and 3D pCASL. 3D-pCASL images were acquired by using a spiral fast spin echo sequence (TR/TE, $4599 \mathrm{~ms} / 9.8 \mathrm{~ms}$; matrix = $512 \times 512 ; \mathrm{FOV}=240 \times 240 \mathrm{~mm}$; number of excitations, 3 ; slice thickness, $4 \mathrm{~mm}$; and post-labeling delay time, $1525 \mathrm{~ms})$.

\section{Image analysis}

All ASL data was transferred to the AW4.6 workstation (GE Healthcare) to generate CBF maps by using GE functool 4.6 software. The calculation of the CBF was

based on the following equation [24], CBF $[\mathrm{mL} / 100 \mathrm{~g} /$ $\min ]=\frac{6000 \times \lambda \times\left(S I_{\text {control }}-S I_{\text {label }}\right) \times e^{\frac{P L D}{T_{1, \text { blood }}}}}{2 \times \alpha \times T_{1, \text { blood }} \times S I_{P D} \times\left(1-e^{T_{1, \text { blood. }}}\right)}$

where $\lambda$ (blood-brain partition coefficient) $=0.9 \mathrm{~mL} / \mathrm{g}$, $\mathrm{T}_{1}$, blood at 3.0 Tesla (the longitudinal relaxation time of blood) $=1650 \mathrm{~ms}, \alpha$ (labeling efficiency) for $\mathrm{pCASL}=$ 0.85, $\tau$ (labeling duration) $=1500 \mathrm{~ms}, \mathrm{SI}_{\text {control }}$ and $\mathrm{SI}_{\text {label }}$ are the time-averaged signal intensities in the control and labeled images, $\mathrm{SI}_{\mathrm{PD}}$ is the signal intensity of a proton density-weighted image. CBF map was preprocessed by using statistical parametric mapping (SPM, http:// www.fil.ion.ucl.ac.uk/spm/software/spm12) based on Matlab2013a platform. Voxel-based analysis (VBA) was 
applied in this study and the specific processing was similar to those in the literature $[25,26]$. At first, the CBF map and 3D T1WI map were manually reoriented to Montreal Neurological Institute (MNI) space and centered on anterior commissure for the following segmentation and spatial normalization. The T1WI map was co-registered to CBF image using a ridged-body model [27]. Voxel-to-voxel affine transformation matrix was generated and written into the T1WI map. Then, the T1WI map was segmented into gray matter, white matter, cerebrospinal fluid by using a unified segmentation algorithm [28]. CBF map was further spatially normalized to standard MNI space (resampling voxel size $=2$ $\mathrm{mm} \times 2 \mathrm{~mm} \times 2 \mathrm{~mm}$ ) with the segmentation information. Finally, quality control was conducted to exclude patients with bad normalization. Spatial smoothing was performed for the normalized CBF map to increase the signal-to-noise ratio (SNR) with a $4 \mathrm{~mm}$ full-width at half-maximum (FWHM) isotropic Gaussian kernel.

\section{Statistical analysis}

One-way analysis of variance (ANOVA) or $\chi^{2}$-test was used to evaluate the difference in age and gender among the three groups. Two-sample $t$-test was applied to compare the differences in disease duration, HAMA, HAMD, MMSE, and MoCA scores between PD and PPS groups. ANOVA was used to identify significant between-group differences in the CBF map among the three groups. Correction for multiple comparisons was performed using Gaussian Random Field (GRF) correction with a voxel-level threshold at $p<0.01$, and cluster-level $p<$ 0.05. The Anatomical Automatic Labeling (AAL) brain template was used to report brain regions. The CBF of brain regions with statistically significant difference were extracted for each subject. A post-hoc ANOVA with the least significant difference test (LSD) was used to identify the significance of pair-wise group (PD vs. PPS, PD vs. $\mathrm{HC}$, and PPS vs. $\mathrm{HC})$ differences in $\mathrm{CBF}$ value $(p<$ $0.05)$. Receiver operating characteristic (ROC) analysis was used to evaluate the performance of CBF from significant brain regions in differentiation between the PD and PPS groups. Pearson's correlation was performed to evaluate the relationship between $\mathrm{CBF}$ values and clinical scores in PD and PPS groups. A $p$ value of less than 0.05 was considered as statistically significant. Statistical analyses were performed by using the SPSS (version 26.0; SPSS, Chicago, III., USA).

\section{Results}

\section{Characteristics of study population}

The clinical and demographic information of the PD and PPS patients are shown in Table 1 , numerical variables were presented as mean \pm standard deviation. All PPS patients initially developed Parkinson's symptoms, including 5 cases of MSA, 2 cases of PSP, 1 case of CBD, and 8 cases of unclassified PPS. There were no statistical differences in gender and age among the PD, PPS, and HC groups $(p>0.05)$. The PD and PPS groups had a significant difference in disease duration $(p=0.02)$. Compared with PD patients, PPS patients had significantly lower MMSE scores $(p=0.001)$. There were no differences in the HAMA, HAMD, and MoCA scores between PD and PPS patients $(p>0.05)$.

\section{CBF in PD, PPS and HC groups}

$\mathrm{CBFs}$ in seven central regions were significantly different among the PD, PPS, and HC groups (GRF correction, voxel-level threshold $p<0.01$, cluster level threshold $p<$ 0.05 ). The specific locations of the brain regions are listed in Table 2 and Fig. 1. The further post-hoc analysis showed that PD group had a significantly decreased CBF in the left frontal medial orbital gyrus (FG_Med Orb), the left triangle inferior frontal gyrus (IFG_Tri), the left middle frontal gyrus (MFG), the right cerebelum_crus2 and the left caudate nucleus $(\mathrm{CN})$, compared with the $\mathrm{HC}$ group $(p<0.05)$. Besides the above regions, the left supplementary motor area (SMA), the right thalamus showed decreased CBF in the PPS group compared with the HC group $(p<0.05)$. PPS group had lower CBF value in the left IFG_Tri, the left MFG, the left $\mathrm{CN}$, the left SMA, and the right thalamus compared with the PD group $(p<0.05)$.

Table 1 Demographic and clinical characteristics of the participants

\begin{tabular}{lllll}
\hline Characteristics & PD $(n=20)$ & PPS $(n=16)$ & Control $(n=17)$ & $p$-Value \\
\hline Age, years & $56.35 \pm 7.56$ & $59.62 \pm 6.89$ & $54.17 \pm 6.58$ & $0.094^{\mathrm{a}}$ \\
Gender, male/female & $13 / 7$ & $7 / 9$ & $6 / 11$ & $0.174^{\mathrm{b}}$ \\
Disease duration years & $6.40 \pm 4.04$ & $3.50 \pm 2.81$ & $\mathrm{NA}$ & $0.020^{\mathrm{c}}$ \\
HAMA scale & $16.50 \pm 5.92$ & $13.36 \pm 7.85$ & $\mathrm{NA}$ & $0.145^{\mathrm{c}}$ \\
HAMD scale & $18.45 \pm 6.91$ & $16.09 \pm 9.30$ & $\mathrm{NA}$ & $0.546^{\mathrm{c}}$ \\
MMSE scale & $26.05 \pm 3.26$ & $19.25 \pm 8.87$ & $\mathrm{NA}$ & $0.001^{\mathrm{c}}$ \\
MoCA scale $^{\mathrm{c}}$ & $20.55 \pm 4.60$ & $13.41 \pm 7.69$ & $\mathrm{NA}$ & $0.231^{\mathrm{c}}$ \\
\hline
\end{tabular}

Abbreviations: ${ }^{\text {a }}$ One-way ANOVA; ${ }^{b}$ Chi-square $\left(X^{2}\right)$ test; ${ }^{c}$ Two-sample $t$-test; PD, Parkinson's disease; PPS, Parkinsonism-Plus syndrome; HAMA, Hamilton Anxiety Scale; HAMD, Hamilton Depression Scale; MMSE, Mini-Mental State Examination; MoCA, Montreal Cognitive Assessment 
Table 2 CBF in different brain regions among the PD, PPS, and HC groups

\begin{tabular}{|c|c|c|c|c|c|c|c|c|c|c|c|}
\hline \multirow{2}{*}{$\begin{array}{l}\text { Peak location } \\
\text { (AAL-90) }\end{array}$} & \multicolumn{3}{|c|}{ MNI coordinate } & \multirow{2}{*}{$\begin{array}{l}\text { No. of } \\
\text { voxels }\end{array}$} & \multirow{2}{*}{$\begin{array}{l}\text { Peak } \\
\text { F-value }\end{array}$} & \multirow{2}{*}{$\begin{array}{l}\mathrm{HC} \\
\text { Mean/SD }\end{array}$} & \multirow{2}{*}{$\begin{array}{l}\text { PD } \\
\text { Mean/SD }\end{array}$} & \multirow{2}{*}{$\begin{array}{l}\text { PPS } \\
\text { Mean/SD }\end{array}$} & \multirow{2}{*}{$\begin{array}{l}\text { PD-HC } \\
p \text {-value }\end{array}$} & \multirow{2}{*}{$\begin{array}{l}\text { PPS-HC } \\
p \text {-value }\end{array}$} & \multirow{2}{*}{$\begin{array}{l}\text { PPS-PL } \\
p \text {-value }\end{array}$} \\
\hline & $x$ & $y$ & z & & & & & & & & \\
\hline SMA.L & -2 & 24 & 60 & 481 & 3.73 & $43.54 / 11.98$ & $42.59 / 10.87$ & $29.04 / 10.15$ & 0.794 & $<0.001$ & 0.001 \\
\hline THA.R & 1 & -16 & 5 & 313 & 3.74 & $58.83 / 12.32$ & $54.01 / 11.62$ & $42.38 / 12.21$ & 0.229 & $<0.001$ & 0.006 \\
\hline FG_Med_Orb.L & 0 & 64 & -4 & 646 & 3.82 & $57.03 / 11.38$ & $47.94 / 12.05$ & $40.56 / 9.34$ & 0.016 & $<0.001$ & 0.053 \\
\hline IFG_Tri.L & -52 & 20 & 2 & 580 & 3.88 & $46.62 / 4.65$ & $41.28 / 9.29$ & $35.15 / 7.07$ & 0.034 & $<0.001$ & 0.017 \\
\hline MFG.L & -40 & 26 & 40 & 377 & 3.93 & $48.80 / 7.25$ & $41.58 / 11.46$ & $33.62 / 9.67$ & 0.029 & $<0.001$ & 0.018 \\
\hline Cere_Crus2.R & 50 & -66 & -50 & 622 & 4.25 & $31.52 / 10.35$ & $19.45 / 13.53$ & $14.58 / 6.15$ & 0.001 & $<0.001$ & 0.182 \\
\hline CN. L & -10 & 4 & 12 & 457 & 4.56 & $33.61 / 5.34$ & 29.48/4.19 & $24.93 / 6.11$ & 0.020 & $<0.001$ & 0.012 \\
\hline
\end{tabular}

Abbreviations: CBF, cerebral blood flow; AAL, automated anatomical labeling; HC, healthy controls; PD, Parkinson's disease; PPS, Parkinsonism-Plus syndrome; SMA, supplementary motor area; THA, thalamus; FG_Med_Orb, frontal medial orbital gyrus; IFG_Tri, triangle inferior frontal gyrus; MFG, middle frontal gyrus; Cere_Crus2, cerebellum_crus2; CN, caudate nucleus; L (R), left (right) hemisphere; SD, standard deviation

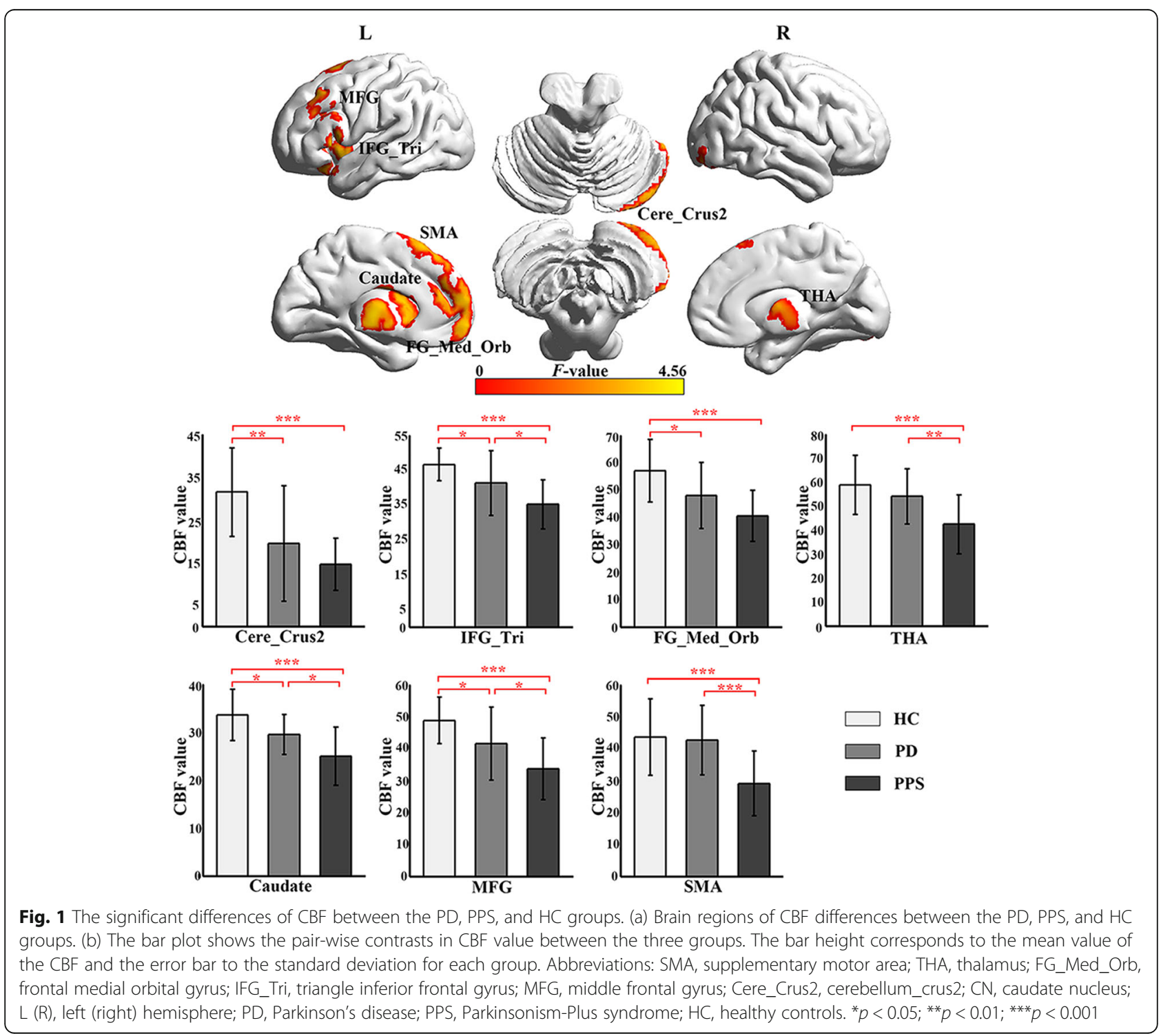


Table 3 ROC analysis of CBF for differentiation between the PD and PPS groups

\begin{tabular}{|c|c|c|c|c|c|c|c|}
\hline \multirow[t]{2}{*}{ Brain region } & \multirow[t]{2}{*}{ AUC } & \multirow[t]{2}{*}{ Cutoff point } & \multirow[t]{2}{*}{ Sensitivity (\%) } & \multirow[t]{2}{*}{ Specificity (\%) } & \multicolumn{2}{|l|}{$95 \% \mathrm{Cl}$} & \multirow[t]{2}{*}{$p$-value } \\
\hline & & & & & Upper bound & Lower bound & \\
\hline SMA.L & 0.831 & 33.723 & 0.800 & 0.750 & 0.696 & 0.967 & 0.001 \\
\hline THA.R & 0.759 & 50.195 & 0.750 & 0.812 & 0.597 & 0.922 & 0.008 \\
\hline CN. L & 0.725 & 24.544 & 0.900 & 0.625 & 0.547 & 0.903 & 0.022 \\
\hline IFG_Tri.L & 0.719 & 39.483 & 0.650 & 0.875 & 0.545 & 0.892 & 0.026 \\
\hline MFG.L & 0.694 & 44.581 & 0.550 & 0.937 & 0.521 & 0.866 & 0.048 \\
\hline
\end{tabular}

Abbreviations: ROC, receiver operating characteristic; PD, Parkinson's disease; PPS, Parkinsonism-Plus syndrome; AUC, area under the curve; Cl, confidence interval; SMA, supplementary motor area; THA, thalamus; CN, caudate nucleus; IFG_Tri, triangle inferior frontal gyrus; MFG, middle frontal gyrus; L (R), left (right) hemisphere

\section{ROC curve analysis}

ROC curve analysis of CBF values in the left MFG, the left IFG_Tri, the left CN, the left SMA, and the right thalamus is shown in Table 3 and Fig. 2. CBF of the left SMA achieved a highest area under the curve (AUC) of $0.831(p=0.001)$, followed by CBF of the right thalamus (AUC: $0.759, p=0.008$ ), CBF of the left CN (AUC: 0.725, $p=0.022$ ), CBF of the left IFG_Tri (AUC: $0.719, p=0.026$ ), and CBF of the left MFG (AUC: 0.694, $p=0.048$ ).

\section{Correlations between CBF values and clinical scores}

The results of correlation analysis are shown in Table 4. The CBFs of left MFG, the left CN, the right thalamus, and the right cerebelum_crus2 were positively correlated with MoCA scores $(r=0.584,0.548,0.723,0.499$, respectively; all $p<0.05)$ and the CBFs of the right thalamus as well as the left $\mathrm{CN}$ were positively correlated with MMSE scores in PD patients $(r=0.745,0.464$, respectively; all $p<0.05)$ in $\mathrm{PD}$ patients. The CBF of left IFG_Tri was positively correlated with the MMSE score $(r=0.783 ; p=0.004)$ and the CBF of

\section{ROC Curves}

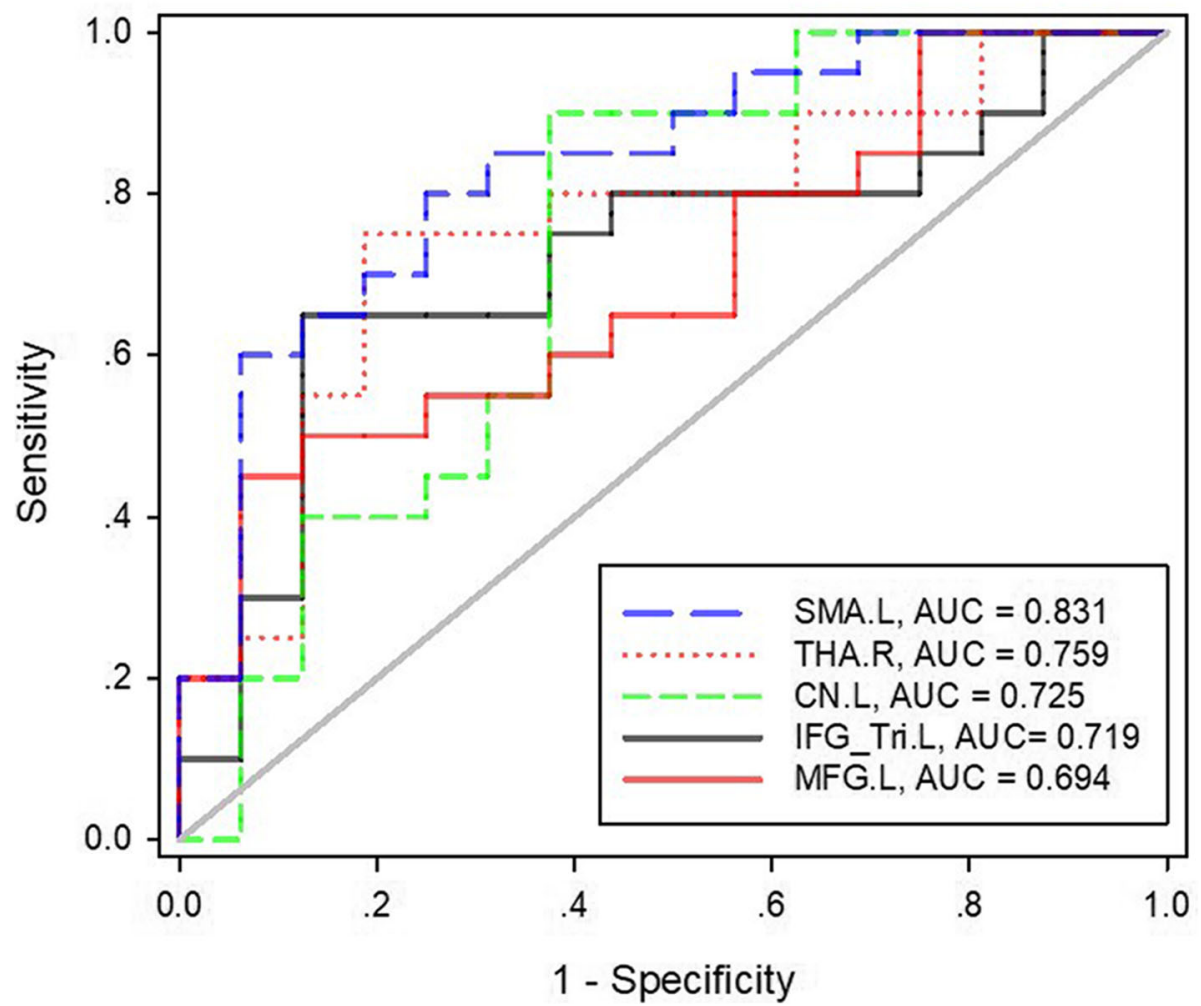

Fig. 2 ROC curves of CBF in the regions with a significant between-group difference for distinguishing PD patients from PPS patients. The diagonal line (grey line) represents the area under the curve of 0.50. Abbreviations: ROC, receiver operating characteristic; SMA, supplementary motor area; THA, thalamus; CN, caudate nucleus; IFG_Tri, triangle inferior frontal gyrus; MFG, middle frontal gyrus; L (R), left (right) hemisphere; PD, Parkinson's disease; PPS, Parkinsonism-Plus syndrome 
Table 4 Correlation between CBF values and clinical scores in PD and PPS groups

\begin{tabular}{|c|c|c|c|c|c|c|c|c|}
\hline & \multicolumn{2}{|l|}{ HAMA } & \multicolumn{2}{|l|}{ HAMD } & \multicolumn{2}{|l|}{ MMSE } & \multicolumn{2}{|c|}{ MoCA } \\
\hline & $r$ & $p$ & $r$ & $p$ & $r$ & $p$ & $r$ & $p$ \\
\hline \multicolumn{9}{|l|}{ PD group } \\
\hline SMA.L & 0.035 & 0.883 & 0.101 & 0.672 & 0.370 & 0.108 & 0.501 & 0.024 \\
\hline THA.R & -0.155 & 0.515 & -0.157 & 0.509 & 0.745 & $<0.001$ & 0.723 & $<0.001$ \\
\hline FG_Med_Orb.L & -0.158 & 0.505 & -0.163 & 0.492 & 0.257 & 0.273 & 0.384 & 0.095 \\
\hline IFG_Tri.L & -0.271 & 0.248 & -0.171 & 0.472 & 0.296 & 0.205 & 0.423 & 0.063 \\
\hline MFG.L & -0.042 & 0.862 & 0.020 & 0.932 & 0.425 & 0.062 & 0.584 & 0.007 \\
\hline Cere_Crus2.R & -0.087 & 0.716 & -0.205 & 0.387 & 0.220 & 0.351 & 0.499 & 0.025 \\
\hline CN.L & -0.024 & 0.920 & -0.032 & 0.894 & 0.464 & 0.039 & 0.548 & 0.012 \\
\hline \multicolumn{9}{|l|}{ PPS group } \\
\hline SMA.L & -0.095 & 0.781 & -0.019 & 0.955 & 0.422 & 0.196 & 0.201 & 0.553 \\
\hline THA.R & 0.053 & 0.878 & 0.299 & 0.372 & 0.267 & 0.427 & 0.179 & 0.599 \\
\hline FG_Med_Orb.L & -0.355 & 0.284 & -0.090 & 0.793 & 0.592 & 0.055 & 0.307 & 0.359 \\
\hline IFG_Tri.L & -0.261 & 0.438 & -0.088 & 0.798 & 0.783 & 0.004 & 0.539 & 0.087 \\
\hline MFG.L & -0.599 & 0.074 & -0.192 & 0.571 & 0.399 & 0.224 & 0.217 & 0.522 \\
\hline Cere_Crus2.R & 0.817 & 0.002 & 0.664 & 0.026 & -0.043 & 0.901 & 0.034 & 0.921 \\
\hline CN.L & -0.218 & 0.520 & -0.061 & 0.859 & 0.376 & 0.255 & 0.105 & 0.759 \\
\hline
\end{tabular}

Abbreviations: CBF, cerebral blood flow; PD, Parkinson's disease; PPS, Parkinsonism-Plus syndrome; SMA, supplementary motor area; THA, thalamus; FG_Med_Orb, frontal medial orbital gyrus; IFG_Tri, frontal inferior, triangle gyrus; MFG, frontal middle gyrus; Cere_Crus2, cerebellum_crus2; CN, caudate nucleus; L (R), left (right) hemisphere; HAMA, Hamilton Anxiety Scale; HAMD, Hamilton Depression Scale; MMSE, Mini-Mental State Examination; MoCA, Montreal Cognitive Assessment

the right cerebellum_crus2 was positively correlated with HAMA and HAMD scores $(r=0.817,0.664$, respectively; all $p<0.05)$ in PPS patients.

\section{Discussion}

Our study results showed that both PD and PPS patients had reduced CBF in several brain regions compared with healthy controls. PPS patients had lower CBF in the left MFG, the left IFG_Tri, the left CN, the left SMA, and the right thalamus than PD patients. CBF value in these five brain regions had a desirable performance for discrimination between PD and PPS.

Previously, arterial spin labeling imaging combined with diffusion tensor imaging has been shown as useful markers for early Parkinson's disease [29]. In our study, PD patients showed reduced $\mathrm{CBF}$ in five brain regions, i.e., the right cerebelum_crus2, the left MFG, the left IFG_Tri, the left FG_ Med_Orb, and the left CN, as detected by 3D-pCASL. These results were consistent with previous studies using PET/SPECT and ASL [23, 30-33], where hypoperfusion was found in widespread cortical regions [20, 23], particularly in frontal regions [23, 30-32], as well as CN [23], cerebellar regions in PD patients [33]. These areas are often associated with motor function, and cognitive impairments or depression in PD [34, 35]. In addition, hypoperfusion in the left $\mathrm{CN}$ and the right cerebelum_crus2 was found in PD patients in our study. Previous studies using ASL technique also found hypoperfusion in the right and left $\mathrm{CN}$ in patients with PD [23] and the CBF laterality pattern in the
$\mathrm{CN}$ was a biomarker for PD diagnosis [36]. The hypoperfusion of $\mathrm{CN}$ maybe relate to the dopamine loss, which is associated with the cognitive decline [37] and depressive symptoms [38] in PD. Our results also showed a positive correlation between the CBFs of the left MFG and the left $\mathrm{CN}$ and non-motor neuropsychological scores including MMSE and MoCA scores in PD patients. This indicated that the CBF reduction might be associated with cognition decline in the PD. The cerebellum is an important component in motor control. To date, contradictory results have been reported for the cerebellum CBF change in the PD, the exact role of the cerebellum in PD remains to be further understood [33].

It is challenging to differentiate the early-middle stage of PPS from PD based on clinical symptoms. Previously, SPECT and PET-CT have been used to discriminate PD from parkinsonian disorders, such as Parkinson variant of MSA and PSP $[39,40]$ based on a distinct hypoperfusion pattern in the frontal cortex, thalamus, and cerebellum [39]. In our study, PPS patients showed reduced CBF in five brain regions similar to those of $\mathrm{PD}$ and in two additional brain regions, i.e., the left SMA and the right thalamus, as detected by 3D-pCASL. Comparatively, PPS patients had altered CBF in more widespread brain regions. The additional involvement of the left SMA and the right thalamus suggests that PPS patients might have more severe impairment of motor function than PD patients. Further correlation analysis showed that the CBF of left IFG_Tri was positively correlated with the MMSE score and the CBF of the right 
cerebellum_crus2 was positively correlated with HAMA and HAMD scores in PPS patients. The association between the hypoperfusion in the cerebellum, thalamus, SMA and the damage in the extrapyramidal system has been described in PPS patients [41]. Thus, more widespread and severe impairment of CBF in PPS might reflect more severity of PPS or later stage of disease.

Our study showed that PPS patients had lower CBF values in the left IFG_Tri, the left MFG, the left CN, the left SMA, and the right thalamus compared with PD patients. This different pattern of reduced brain perfusion between PPS and PD is consistent with the previous study, where the perfusion distribution patterns were found to be different among MSA-P, PSP, and PD [42]. In our study, further ROC analysis showed that CBF value in these five brain regions had a desirable AUC for discrimination between PD and PPS, with CBF of the left SMA achieving the highest AUC of 0.831. Previously, a multitude of imaging parameters have been used to differentiate between PD and PPS [9, 43-45]. Tir et al. found that PSP had a lower fractional anisotropy value derived from diffusion tensor imaging in the SMA [46]. Calloni et al. reported that the middle cerebellar peduncle width and putaminal hypointensity on susceptibilityweighted imaging (SWI) can be used in combination to distinguish atypical parkinsonisms from idiopathic PD, with an AUC $=0.98$ [43]. In addition, swallow tail (AUC = $0.85)$ and putaminal hypointensity $(\mathrm{AUC}=0.68)$ also were reported to be able to distinguish MSA from PD [9]. Comparatively, the participants in these studies were older and had longer disease duration. In our study, PPS patients in the earlier stage of the disease were included. Our results showed the CBF of the SMA had the greatest AUC (0.831) and might be used as a surrogate marker for the differential diagnosis between the two diseases in an earlier stage.

There are some limitations to this study. First, we did not investigate $\mathrm{CBF}$ in early PD patients. The CBF as detected by ASL has been reported in early PD patients and perfusion reduction did not differ among different stages of PD [29]. Second, the sample size is small. Third, the numbers of clinical scales collected are insufficient. Specifically, Unified Parkinson's Disease Rating Scale (UPDRS) as a rating tool to assess motor symptoms of PD patients was not applied in our study to evaluate the motor symptoms in PPS patients. Thus, motor clinical scores were not available for all patients. Third, a single PLD time was used in our study. Although the PLD of $1525 \mathrm{~ms}$ was commonly used in previous ASL studies [20,47]. However, a fixed PLD could bring the risk of inaccurate estimation of the CBF when the PLD used is shorter or longer than the arterial transit time. Future studies with multiple PLDs are needed to validate the discriminative $\mathrm{CBF}$ in these two diseases.
Finally, CBF was measured in each patient under medication in "on" state. The effect of medication state on CBF requires further investigation.

\section{Conclusion}

In conclusion, our study demonstrated that PD and PPS patients showed hypoperfusion in several brain regions and PPS showed severer and more widespread impairment in CBF. PD and PPS patients have a certain discriminative pattern of reduced $\mathrm{CBFs}$, in particular, reduced CBF in left SMA, which can be used as a surrogate marker for differential diagnosis.

\section{Abbreviations \\ PD: Parkinson's disease; PPS: Parkinsonism-Plus syndrome; MSA: Multiple system atrophy; PSP: Progressive supranuclear palsy; CBD: Corticobasal degeneration; MRI: Magnetic resonance imaging; PET: Positron emission tomography; SPECT: Single-photon emission computed tomography; ASL: Arterial spin labeling; 3D-pCASL: Three-dimensional pseudo-continuous arterial spin labeling; CBF: Cerebral blood flow; HCs: Healthy controls; HAMA: Hamilton Anxiety Scale; HAMD: Hamilton Depression Scale; MMSE: Mini-Mental State Examination; MoCA: Montreal Cognitive Assessment; TR: Repetition time; TE: Echo time; TI: Inversion time; FA: Flip angel; FOV: Field of view; VBA: Voxel-based analysis; MNI: Montreal Neurological Institute; SNR: Signal-to-noise ratio; FWHM: Full-width at half- maximum; ANOVA: One-way analysis of variance; GRF: Gaussian Random Field; AAL: Anatomical Automatic Labeling; LSD: Least significant difference test; ROC: Receiver operating characteristic; FG_Med_Orb: Frontal medial orbital gyrus; IFG_Tri: Triangle inferior frontal gyrus; MFG: Middle frontal gyrus; CN: Caudate nucleus; SMA: Supplementary motor area; AUC: Area under the curve; SWI: Susceptibility-weighted imaging; PLD: Post-labeling delay}

\section{Acknowledgements}

Not applicable.

\section{Authors' contributions}

LC, XW, and JS designed the study. LC, YW, WW, PH, and HL collected the data. LC, XW, RG, YW, WW, PH, and HL analyzed and interpreted the data. LC, and XW wrote the article. JS revised the article. The authors read and approved the final manuscript.

\section{Funding}

The study collection of data was supported by the Medical Science and Technology Research Fund in Guangdong Province of China (B2019111).

\section{Availability of data and materials}

The datasets generated and/or analysed during the current study are available from the corresponding author on reasonable request.

\section{Ethics approval and consent to participate}

This study was approved by the Ethics Committee of Guangdong 999 Brain Hospital (Guangzhou, China), and written informed consent was obtained from all participants.

\section{Consent for publication}

Not applicable.

\section{Competing interests}

The authors declare that they have no competing interests.

\section{Author details}

${ }^{1}$ Department of Radiology, Sun Yat-Sen Memorial Hospital, Sun Yat-Sen University, Guangzhou 510120, China. ²Department of Radiology, Guangdong 999 Brain Hospital, Guangzhou 510510, China. ${ }^{3}$ Department of Radiology, The Third Affiliated Hospital, Sun Yat-Sen University, Guangzhou 510630, China. ${ }^{4}$ Department of Neurology, Guangdong 999 Brain Hospital, Guangzhou 510510, China. 


\section{Received: 12 January 2020 Accepted: 6 July 2020}

\section{Published online: 13 July 2020}

\section{References}

1. Davie CA. A review of Parkinson's disease. Br Med Bull. 2008:86:109-27.

2. Brooks DJ. Parkinson's disease: diagnosis. Parkinsonism Relat Disord. 2012; 18(Suppl 1):S31-3.

3. Mitra K, Gangopadhaya PK, Das SK. Parkinsonism plus syndrome--a review. Neurol India. 2003:51(2):183-8.

4. Srivanitchapoom P, Pitakpatapee $Y$, Suengtaworn A. Parkinsonian syndromes: A review. Neurol India. 2018;66(Supplement):S15-25.

5. Wenning GK, Colosimo C. Diagnostic criteria for multiple system atrophy and progressive supranuclear palsy. Rev Neurol (Paris). 2010;166(10):829-33.

6. Orimo S. New development of diagnosis and treatment for Parkinson's disease. Rinsho Shinkeigaku. 2017;57(6):259-73.

7. Orimo S. Initial diagnosis of Parkinson's disease - neuroradiological diagnosis. Rinsho Shinkeigaku. 2013;53(11):977-80.

8. Bacchi S, Chim I, Patel S. Specificity and sensitivity of magnetic resonance imaging findings in the diagnosis of progressive supranuclear palsy. J Med Imaging Radiat Oncol. 2018;62(1):21-31.

9. Wang N, Yang H, Li C, Fan G, Luo X. Using 'swallow-tail' sign and putaminal hypointensity as biomarkers to distinguish multiple system atrophy from idiopathic Parkinson's disease: a susceptibility-weighted imaging study. Eur Radiol. 2017:27(8):3174-80.

10. Yeo T, Tan LC. 'Hummingbird' sign in a patient with Guam parkinsonismdementia complex. J Mov Disord. 2017;10(3):145-8.

11. Sakurai K, Tokumaru AM, Shimoji K, Murayama S, Kanemaru K, Morimoto S, et al. Beyond the midbrain atrophy: wide spectrum of structural MRI finding in cases of pathologically proven progressive supranuclear palsy. Neuroradiology. 2017:59(5):431-3.

12. Yagi S, Yoshikawa E, Futatsubashi M, Yokokura M, Yoshihara Y, Torizuka T, et al. Progression from unilateral to bilateral parkinsonism in early Parkinson disease: implication of mesocortical dopamine dysfunction by PET. J Nucl Med. 2010;51(8):1250-7.

13. Uyama N, Otsuka H, Shinya T, Otomi $Y$, Harada M, Sako W, et al. The utility of the combination of a SPECT study with [123I]-FP-CIT of dopamine transporters and [123I]-MIBG myocardial scintigraphy in differentiating Parkinson disease from other degenerative parkinsonian syndromes. Nucl Med Commun. 2017;38(6):487-92

14. Chelban V, Bocchetta M, Hassanein S, Haridy NA, Houlden H, Rohrer JD. An update on advances in magnetic resonance imaging of multiple system atrophy. J Neurol. 2019;266(4):1036-45.

15. Tsuda M, Asano S, Kato Y, Murai K, Miyazaki M. Differential diagnosis of multiple system atrophy with predominant parkinsonism and Parkinson's disease using neural networks. J Neurol Sci. 2019;401:19-26.

16. Orimo S. Diagnostic imaging in Parkinson's disease. Nihon Rinsho. 2017; 75(1):48-55.

17. Stezin A, Lenka A, Jhunjhunwala K, Saini J, Pal PK. Advanced structural neuroimaging in progressive supranuclear palsy: where do we stand? Parkinsonism Relat Disord. 2017:36:19-32.

18. Dabrowska M, Schinwelski M, Sitek EJ, Muraszko-Klaudel A, Brockhuis B, Jamrozik Z, et al. The role of neuroimaging in the diagnosis of the atypical parkinsonian syndromes in clinical practice. Neurol Neurochir Pol. 2015; 49(6):421-31.

19. Dolui S, Vidorreta M, Wang Z, Nasrallah IM, Alavi A, Wolk DA, et al. Comparison of PASL, PCASL, and background-suppressed 3D PCASL in mild cognitive impairment. Hum Brain Mapp. 2017;38(10):5260-73.

20. Melzer TR, Watts R, MacAskill MR, Pearson JF, Rüeger S, Pitcher TL, et al, Arterial spin labelling reveals an abnormal cerebral perfusion pattern in Parkinson's disease. Brain. 2011;134(Pt 3):845-55.

21. Zheng W, Ren S, Zhang H, Liu M, Zhang Q, Chen Z, et al. Spatial patterns of decreased cerebral blood flow and functional connectivity in multiple system atrophy (cerebellar-type): a combined arterial spin labeling perfusion and resting state functional magnetic resonance imaging study. Front Neurosci. 2019;13:777.

22. Li J, Jin M, Wang L, Qin B, Wang K. MDS clinical diagnostic criteria for Parkinson's disease in China. J Neurol. 2017;264(3):476-81.

23. Fernández-Seara MA, Mengual E, Vidorreta M, Aznárez-Sanado M, Loayza FR, Villagra F, et al. Cortical hypoperfusion in Parkinson's disease assessed using arterial spin labeled perfusion MRI. Neurolmage. 2012;59(3):2743-50.
24. Alsop DC, Detre JA, Golay X, et al. Recommended implementation of arterial spin-labeled perfusion MRI for clinical applications: a consensus of the ISMRM perfusion study group and the European consortium for ASL in dementia. Magn Reson Med. 2015:73(1):102-16.

25. Denier N, Gerber H, Vogel M, Klarhöfer M, Riecher-Rossler A, Wiesbeck GA, et al. Reduction in cerebral perfusion after heroin administration: a resting state arterial spin labeling study. PLoS One. 2013;8(9):e71461.

26. Mersov AM, Crane DE, Chappell MA, Black SE, Maclntosh BJ. Estimating the sample size required to detect an arterial spin labelling magnetic resonance imaging perfusion abnormality in voxel-wise group analyses. J Neurosci Methods. 2015:245:169-77.

27. Collignon A, Maes F, Delaere D, et al. Automated multi-modality image registration based on information theory. In: Bizais Y, Barillot C, Di Paola R, editors. Information processing in medical imaging. Dordrecht: Kluwer Academic Publishers; 1995. p. 263-74.

28. Ashburner J, Friston KJ. Unified segmentation. Neuroimage. 2005;26(3):839-51.

29. Wei X, Yan R, Chen Z, Weng R, Liu X, Gao H, et al. Combined diffusion tensor imaging and arterial spin labeling as markers of early Parkinson's disease. Sci Rep. 2016;6:33762.

30. Paschali A, Messinis L, Lyros E, Constantoyannis C, Kefalopoulou Z, Lakiotis V, et al. Neuropsychological functions and rCBF SPECT in Parkinson's disease patients considered candidates for deep brain stimulation. Eur J Nucl Med Mol Imaging. 2009;36(11):1851-8.

31. Firbank MJ, Molloy S, McKeith IG, Burn DJ, O'Brien JT. Longitudinal change in 99mTcHMPAO cerebral perfusion SPECT in Parkinson's disease over one year. J Neurol Neurosurg Psychiatry. 2005;76(10):1448-51.

32. Antonini A, De Notaris R, Benti R, De Gaspari D, Pezzoli G. Perfusion ECD/ SPECT in the characterization of cognitive deficits in Parkinson's disease. Neurol Sci. 2001;22(1):45-6.

33. Song IU, Chung YA, Chung SW, Jeong J. Early diagnosis of Alzheimer's disease and Parkinson's disease associated with dementia using cerebral perfusion SPECT. Dement Geriatr Cogn Disord. 2014;37(5-6):276-85.

34. Kim YD, Jeong HS, Song IU, Chung YA, Namgung E, Kim YD. Brain perfusion alterations in depressed patients with Parkinson's disease. Ann Nucl Med. 2016;30(10):731-7

35. Lin WC, Chen PC, Huang CC, Tsai NW, Chen HL, Wang HC, et al. Autonomic function impairment and brain perfusion deficit in Parkinson's disease. Front Neurol. 2017:8:246.

36. Yamashita K, Hiwatashi A, Togao O, Kikuchi K, Yamaguchi H, Suzuki Y, et al. Cerebral blood flow laterality derived from arterial spin labeling as a biomarker for assessing the disease severity of parkinson's disease. J Magn Reson Imaging. 2017;45(6):1821-6.

37. Niethammer M, Tang CC, Ma Y, Mattis PJ, Ko JH, Dhawan $V$, et al. Parkinson's disease cognitive network correlates with caudate dopamine. Neuroimage. 2013;78:204-9.

38. Vriend $C$, Raijmakers $P$, Veltman DJ, van Dijk KD, van der Werf YD, Foncke $E M$, et al. Depressive symptoms in Parkinson's disease are related to reduced [123I]FP-CIT binding in the caudate nucleus. J Neurol Neurosurg Psychiatry. 2014;85(2):159-64.

39. Kimura N, Hanaki S, Masuda T, Hanaoka T, Hazama Y, Okazaki T, et al. Brain perfusion differences in parkinsonian disorders. Mov Disord. 2011;26(14): 2530-7.

40. Hong CM, Ryu HS, Ahn BC. Early perfusion and dopamine transporter imaging using ${ }^{18} \mathrm{~F}-\mathrm{FP}-\mathrm{CIT}$ PET/CT in patients with parkinsonism. Am J NuCl Med Mol Imaging. 2018:8(6):360-72.

41. Höglinger GU, Kassubek J, Csoti I, Ehret R, Herbst H, Wellach I, et al. Differentiation of atypical Parkinson syndromes. J Neural Transm (Vienna). 2017;124(8):997-1004.

42. Murakami N, Sako W, Haji S, Furukawa T, Otomi Y, Otsuka H, et al. Differences in cerebellar perfusion between Parkinson's disease and multiple system atrophy. J Neurol Sci. 2019;409:116627.

43. Calloni SF, Conte G, Sbaraini S, Cilia R, Contarino VE, Avignone S, et al. Multiparametric MR imaging of Parkinsonisms at 3 tesla: its role in the differentiation of idiopathic Parkinson's disease versus atypical Parkinsonian disorders. Eur J Radiol. 2018;109:95-100.

44. Sako W, Abe T, Murakami N, Miyazaki Y, Izumi Y, Harada M, et al. Imagingbased differential diagnosis between multiple system atrophy and Parkinson's disease. J Neurol Sci. 2016:368:104-8.

45. Brajkovic L, Kostic V, Sobic-Saranovic D, Stefanova E, Jecmenica-Lukic M, Jesic $A$, et al. The utility of FDG-PET in the differential diagnosis of parkinsonism. Neurol Res. 2017;39(8):675-84. 
46. Tir M, Delmaire C, Besson P, Defebvre L. The value of novel MRI techniques in Parkinson-plus syndromes: diffusion tensor imaging and anatomical connectivity studies. Rev Neurol (Paris). 2014;170(4):266-76.

47. Le Heron CJ, Wright SL, Melzer TR, et al. Comparing cerebral perfusion in Alzheimer's disease and Parkinson's disease dementia: an ASL-MRI study. J Cereb Blood Flow Metab. 2014;34(6):964-70.

\section{Publisher's Note}

Springer Nature remains neutral with regard to jurisdictional claims in published maps and institutional affiliations.

Ready to submit your research? Choose BMC and benefit from:

- fast, convenient online submission

- thorough peer review by experienced researchers in your field

- rapid publication on acceptance

- support for research data, including large and complex data types

- gold Open Access which fosters wider collaboration and increased citations

- maximum visibility for your research: over $100 \mathrm{M}$ website views per year

At BMC, research is always in progress.

Learn more biomedcentral.com/submissions 